\title{
L-Tryptophan in Infusionslösungen
}

Das Bundesgesundheitsamt hat die Zulassung verschiedener tryptophanhaltiger Präparate ausgesetzt. Dies gilt nur für oral anzuwendende Präparate mit L-Tryptophan unterschiedlicher Indikationen. Anlaß zu dieser Verfügung gaben mehrere Be-obachtungen, daß nach längerer Anwendung von oral zuzu-führenden tryptophanhaltigen Präparaten Nebenwirkungen auftraten. Von dieser Verfügung des Bundesgesundheitsamtes sind tryp-tophanhaltige Infusionslösungen nicht betroffen. Tryptophan ist eine essentielle Aminosäure und muß in Aminosäurelösun-gen für eine vollwertige parenterale Ernährung in bedarfsdek-kenden Mengen enthalten sein. Aminosäurelösungen mit zu wenig Tryptophan oder ganz ohne Tryptophan könnten das therapeutische Ziel einer Deckung des Eiweißbedarfs nicht erfüllen. Daraus entstehende Imbalanzen könnten den Patienten sogar Schaden zufügen. Deshalb ist bei einer Indikation zur künstlichen Ernährung auch die Verwendung tryptophanhaltiger Aminosäurelösungen notwendig. Ergänzend dazu ist festzustellen, daß bei Verwendung von Aminosäurelösungen mit einem bedarfsdeckenden Gehalt an Tryptophan bisher keine Nebenwirkungen beobachtet worden sind. An die Qualität von Rohstoffen für die Herstellung von Infusionslösungen werden besonders hohe Ansprüche gestellt. Patienten, die aus verschiedenen Gründen ihren Nährstoffbe-darf auf normalem Weg nicht mehr decken können, müssen künstlich ernährt werden. Für die vollwertige künstliche Ernährung, ob parenteral oder enteral, ist die Verwendung einer bedarfsdeckenden Menge von Tryptophan unbedingt notwendig. Ausschuß für Nährstoffbedarf der DAKE

Buchbesprechungen · Book Reviews Infusionstherapie 1990;17:352

Sobotta-Atlas der Anatomie

Herausgeber: J. Staubesand

Urban \& Schwarzenberg, München 1988

Band 1 - Kopf, Hals, Obere Extremitäten:

415 S. ,672 größtenteils farbige Abb. , Kunststoff, DM 120, ISBN 3-541-02819-X

Band 2 - Bauch, Becken, Untere Extremitäten: 379 S. , 604 teils farbige Abb. , Kunststoff, DM 120,-

ISBN 3-541-02829-7

Gleich, um welche Auflage es sich handelt, der «Sobotta» ist wohl nach wie vor weltweit das Standardwerk in der Anatomie, für Studenten unentbehrlich, und später, notwendiges Nachschlage werk.

Die jetzt vorliegende 19. Auflage hat beim oberflächlichen Durchblättern zwei ins Auge stechende Neuerungen: Die Farben der einzelnen Abbildungen sind leuchtender, das bedrückende Grün-Grau ist verschwunden. Und als zweiter Punkt wurde ein neues gerades und modernes Schriftbild gewählt, was im Zeitalter der Computerschrift wesentlich einprägsamer ist. Durch eine neue, unterschiedliche Farbge-bung 
von Muskeln, Geweben etc., sind die anatomischen Verhältnisse der einzelnen Regionen besser zu erkennen, die Legenden unter den Abbildungen sind ausführlicher gewor-den und damit genauer. Alle Darstellungen und technischen Untersuchungsmethoden wurden in der Wiedergabe entschei-dend verbessert; Sonogramme, wichtig im Vergleich zu den gemalten anatomischen Verhältnissen, sind neu dazugekom-men.

Für Studenten sicher ganz wichtig ist die übersichtlichere, sehr klare und damit schnell zu erfassende tabellarische Zusam-menstellung von Muskeln, Knochen, Nerven und deren Funktionen am Ende eines jeden Kapitels.

Die 19. Auflage des «Sobotta» ist somit ein Klassiker der medizinischen Standardwerke, aber auf dem neuesten technischen und wissenschaftlichen Stand.

M. Fischer, Grünwald 